\title{
Genetic variability among Fusarium oxysporum isolates from melon (Cucumis melo) in Qazvin province, Iran
}

\author{
Mohammadreza Mahdikhani* \\ M.Sc Graduated of Plant Pathology, Department of Plant Pathology, Bu-Ali Sina University, Hamedan, Iran
}

\author{
Received: 28.12 .2015 \\ Revised: 08.02.2016 \\ Accepted: 03.03.2016 \\ Published: 25.04.2016 \\ *Address for \\ correspondence: \\ Mohammadreza \\ Mahdikhani, \\ M.Sc Graduated of Plant \\ Pathology, Department of \\ Plant Pathology, Bu-Ali Sina \\ University, Hamedan, Iran. \\ Tel: +98-9380984269, \\ E-mail:m.mahdikhani2016@ \\ gmail.com
}

\begin{abstract}
Fusarium wilt, caused by Fusarium oxysporum f. sp. melonis (Fom), the soilborne fungus that can due to more losses in yield of melon plants. Fusarium is a variable fungus in terms of morphology, symptom production, pathogenicity, and wide host range. From 2013 to 2015, 120 isolates of $F$. oxysporum, Fusarium acuminatum, Fusarium graminearum, Fusarium proliferatum, and Fusarium solani from melon were collected from disease suspected plants from the field and defined for pathogenicity in our melon plants. Then, a sort of those was elected for phylogenetic analysis. In the beginning, the Fusarium isolates were classified based on morphology and identifications were authenticated based on sequence data from elongation factor $1 \alpha$ and DNA and then were used to determine whether Fusarium variability is relevant to geographic origin and pathogenicity. Neighborjoining analyses datasets indicated some clades based on geographic origin, but there is not even a single clades including solely of pathogens. Because of the factors affecting in pathogenicity are variable, we should be considered them in future studies. Due to the presence of Fom and some nonpathogenic isolates in almost all clades, it is obvious that Fom is not monophyletic.
\end{abstract}

KEY WORDS: Fusarium wilt, neighbor-joining, pathogenicity, phylogenetic

\section{INTRODUCTION}

Fusarium wilt is a soilborne disease which is the result of Fusarium oxysporum Schl. This fungi is one of the most important pathogens, which is causes wilting in some crops such as cantaloupe, cucumber, wax gourd, muskmelon, watermelon, and other types of melon. Melon (Cucumis melo) of the Cucurbitaceae family is one of the most important agricultural products in Qazvin Province, Iran. Fusarium is a soilborne fungus that is found in all soils and has global distribution. All isolates are able to survive in soil and growth in the rhizosphere area of many plant species. In the previous reports, more than 120 different formae speciales of F.oxysporum have been described based on host specificity (Armstrong and Armstrong, 1981). Wilting symptoms are the result of fungal spores and mycelium that block the xylem. Infected plants looking healthy at the beginning, but the vascular tissue is brown and discolored. To support morphological identification of Fusarium species, we can use of diversity in DNA sequences of genes. Furthermore, to estimate the genetic relationship for F. oxysporum we can use of phylogenetic analysis on DNA sequences (Hsuan et al., 2011; Harrow et al., 2010). Genetic diversity of a particular "forma specialis" revealed by using of multitude ways. Those include restriction fragment length polymorphism (Koenig et al., 1997), random amplified polymorphic DNA (RAPD) (Windels, 1992), amplified fragment length polymorphism )Leslie et al., 2006). There aren't any affective fungicides for controlling this pathogen on melon. Furthermore, crop rotation is not effective because isolates on the melon plant can live on the other crops (Gordon et al., 1989). Hence, host resistance is the best way for disease management. To host plant resistance breeding, we must know if exists the variability within the pathogen population. Therefore, the present research study was performed to explain variation among F. oxysporum isolated from melon using DNA sequencing of translation elongation factor $1 \alpha$ (TEF $1 \alpha)$. The goal of this study was to determine phylogenetic relationship among F. oxysporum isolates, gathered from melon plants, and determine diversity correlates with location origin and pathogenicity. 


\section{MATERIALS AND METHODS}

In recent years, prevalence a disease had caused a severe decreasing in melon yield in Qazvin Province, Iran (Middle of Iran, $\left.36^{\circ} 15^{\prime} \mathrm{N}-50^{\circ} 0^{\prime} \mathrm{E}\right)$. Disease symptoms in melon farmlands were similar to Fusarium wilt. F. oxysporum consists of pathogenic and non-pathogenic strains that are morphologically indistinguishable.

\section{Sampling, Identification, and Storage}

Melon plants symptoms of Fusarium wilt were elected from melon farmland of Qazvin province from 2013 to 2015. Each sample was rinsed under running water for $20 \mathrm{~min}$. Six pieces from root, crown, and stem $(5 \mathrm{~mm})$ were surface sterilized with $2 \% \mathrm{NaCl}_{2}$ for $2 \mathrm{~min}$. These pieces were cultured onto potato dextrose agar (PDA; $200 \mathrm{~g}$ potato, $17 \mathrm{~g}$ glucose and $17 \mathrm{~g}$ agar/L) supplemented with 1 drop lactic acid. Petri dishes were incubated at $25^{\circ} \mathrm{C}$ for 10 days under fluorescent light (12 h photoperiod) (Nirenberg, 1976). Morphological identification was done on PDA and carnation leaf agar medium according to the morphological criteria keys of Nelson et al. (1983). For long-time storage, mycelium from $F$ oxysporum was stored desiccated on sterile glass fiber filter paper at $-18^{\circ} \mathrm{C}$ (Hanson and Hill, 2004).

\section{Pathogenicity Tests}

A total of 120 Fusarium isolates cultured from diseased melon, tomato and watermelon were surveyed for pathogenicity on a Fusarium wilt susceptible melon plant Minoo 095P ergon (Ergon International N.V. The Netherlands). All isolates of Fusarium were grown in $250 \mathrm{ml}$ volume conical flask, containing $100 \mathrm{ml}$ Armstrong broth which is used for mass multiplication of inoculum of Fusarium (Booth, 1971). The flasks were incubated at $27^{\circ} \mathrm{C}$, 120 rpm for 6 days into an incubator shaker. After 6 days, all cultures of Fusarium isolates were filtered through a muslin cloth before adjusting the spore density with distilled water to $2 \times 10^{7}$ spores / g of soil mixture in pots. Melon seedlings variety Minoo 095P was raised in steam sterilized soil. 21 days seedlings were transferred into the root trainers. The experiment was carried out in a greenhouse at $27^{\circ} \mathrm{C}$ and $13 \mathrm{~h}$ light period (600 lux). Plants were irrigated as and when required and supplied with Agrimel's solution weekly. Four replications were maintained for every isolate of $F$ oxysporum. The disease incidence was measured after 4 weeks of transplantation (Joshi et al., 2013). All tests were conducted at least twice. Disease severity was recorded using a scale containing 4 grades suggested by Matsumoto et al. (2011): Grade: $0=$ No symptoms, $1=$ Small lesions on leaves, $2=$ Leaves strongly affected, $3=$ Plant death. After 4 weeks, roots and crown were removed to reisolate and confirmed the presence of Fusarium species. Disease index (DI) values were analyzed using PROC MIXED (SAS) with $t$-test (Dunnett, 1955), to determine if there were significant differences among our isolates or not. Half $(50 \%)$ of the isolates from different locations and pathogenicity were selected for phylogenetic analysis (Hill et al., 2011) (Table 1).

\section{DNA Extraction from Fungal Cultures}

Each fungal culture was inoculated in liquid potato dextrose broth and grown on a rotary shaker at $24^{\circ} \mathrm{C}$ for 5 days $100 \mathrm{rpm}$ with $10 \mathrm{~h}$ of light per day and aeration. The fungal mycelium was harvested, ground to fine powder in liquid nitrogen. Genomic DNA was extracted from each culture by cetyltrimethylammonium bromide method. DNA samples were dried under vacuum and dissolved in TE buffer (10 mM Tris-HCl, $1 \mathrm{mM}$ EDTA, $\mathrm{pH} 8.0$ ), followed by incubation with RNase $\mathrm{A}$ at a concentration of $100 \mu \mathrm{g} / \mathrm{mL}$ at $37^{\circ} \mathrm{C}$ for $1 \mathrm{~h}$ (Gurjar et al., 2009).

\section{DNA Amplification}

We used an intron region of TEF $1 \alpha$, to describe in $F$. oxysporum isolated from diseased melon plants. The primer used was described initially by O'Donnell et al. (1998) for EF1 $\alpha$. Amplification reactions were performed in volumes of $25 \mu \mathrm{l}$ containing $1.5 \mu \mathrm{l}$ of genomic DNA (25 mg), $1.5 \mu \mathrm{l}$ of $\times 10$ buffer polymerase chain reaction (PCR) (100 mM Tris- $\mathrm{HCl}, 15 \mathrm{mM} \mathrm{MgCl}, 500 \mathrm{mM}$ $\mathrm{KCl}, \mathrm{pH} 8), 1 \mu \mathrm{l}$ of $\mathrm{Mg} \mathrm{Cl} 2(50 \mathrm{mM}), 0.25 \mu \mathrm{l}$ of dNTPs $(100 \mathrm{mM}), 0.2 \mu \mathrm{l}$ of Taq DNA polymerase $(5 \mathrm{U} / \mathrm{ml})$, and $25 \mu \mathrm{l}$ of each primer $(20 \mathrm{mM})$. All reactions in our study were repeated at least twice.

\section{Sequencing and Phylogenetic Analysis}

The PCR products were purified using a Gene Elute PCR clean-up kit (Sigma, USA) pursuant to the manufacturer's protocol. The purified PCR products sent for sequencing to a service provider. The EF1 $\alpha$ sequences were aligned with clustalx-2.1 (Thompson et al., 1997). Then, default gap penalties were used in the pair-wise alignment. A neighbor-joining tree was delineated with the JukesCantor model. The reliability of neighbor-joining trees was calculated by bootstrap method with 1000 random stepwise along with replicates. An isolate of Fusarium culmorum was selected as the outgroup.

\section{RESULTS}

\section{Species Identification}

Based on the morphological characteristics of isolates from farmlands, we identified F. oxysporum, Fusarium acuminatum, 
Table 1: Geographic origin and pathogenicity on melon seedling (variety Minoo 095P) of Fusarium oxysporum, Fusarium acuminatum, Fusarium graminearum, Fusarium proliferatum and Fusarium solani cultured from melon, tomato and watermelon

\begin{tabular}{|c|c|c|c|c|c|}
\hline Isolatea & Location & $\begin{array}{l}\text { Morphological } \\
\text { identification }\end{array}$ & Host & $\begin{array}{l}\text { Year of } \\
\text { isolation }\end{array}$ & Pathogenicity \\
\hline F14 & Takestan & Oxysporum & Melon & 2013 & NP \\
\hline F16 & Takestan & Oxysporum & Melon & 2013 & NP \\
\hline F22 & Takestan & Oxysporum & Melon & 2015 & NP \\
\hline F23 & Takestan & Oxysporum & Melon & 2015 & NP \\
\hline R12 & Buein zahra & Oxysporum & Melon & 2013 & $\mathrm{P}$ \\
\hline R15 & Buein zahra & Oxysporum & Melon & 2013 & $P$ \\
\hline R16 & Buein zahra & Oxysporum & Melon & 2013 & $P$ \\
\hline R18 & Buein zahra & oxysporum & Melon & 2013 & P \\
\hline R20 & Buein zahra & Oxysporum & Melon & 2015 & $P$ \\
\hline R22 & Buein zahra & Oxysporum & Melon & 2015 & $\mathrm{P}$ \\
\hline R27 & Buein zahra & Oxysporum & Melon & 2015 & LP \\
\hline R30 & Buein zahra & Acuminatum & Melon & 2015 & LP \\
\hline R32 & Buein zahra & Acuminatum & Melon & 2015 & LP \\
\hline K6 & Choobindar & Oxysporum & Melon & 2013 & $P$ \\
\hline K7 & Choobindar & Oxysporum & Melon & 2013 & $P$ \\
\hline K8 & Choobindar & Oxysporum & Melon & 2013 & $P$ \\
\hline Kl1 & Choobindar & Oxysporum & Melon & 2013 & $\mathrm{P}$ \\
\hline K12 & Choobindar & Oxysporum & Melon & 2015 & LP \\
\hline K15 & Choobindar & Solani & Melon & 2015 & NP \\
\hline S1 & Sagez abad & Oxysporum & Melon & 2013 & $\mathrm{P}$ \\
\hline S2 & Sagez abad & Oxysporum & Melon & 2013 & $P$ \\
\hline S4 & Sagez abad & Oxysporum & Watermelon & 2014 & NP \\
\hline S6 & Sagez abad & Oxysporum & Watermelon & 2014 & NP \\
\hline S9 & Sagez abad & Oxysporum & Watermelon & 2015 & NP \\
\hline S10 & Sagez abad & Acuminatum & Melon & 2015 & LP \\
\hline D14 & Danesfahan & Oxysporum & Melon & 2013 & $\mathrm{P}$ \\
\hline D15 & Danesfahan & Oxysporum & Tomato & 2013 & $P$ \\
\hline D19 & Danesfahan & Oxysporum & Tomato & 2013 & LP \\
\hline D29 & Danesfahan & Oxysporum & Tomato & 2014 & $\mathrm{P}$ \\
\hline D33 & Danesfahan & Oxysporum & Tomato & 2014 & $P$ \\
\hline D37 & Danesfahan & Solani & Melon & 2015 & NP \\
\hline D41 & Danesfahan & Graminearum & Melon & 2015 & NP \\
\hline Alo & Abyek & Oxysporum & Melon & 2013 & $P$ \\
\hline All & Abyek & Oxysporum & Melon & 2013 & $P$ \\
\hline Al2 & Abyek & Oxysporum & Melon & 2013 & $\mathrm{P}$ \\
\hline Al5 & Abyek & Oxysporum & Melon & 2014 & LP \\
\hline Al6 & Abyek & Oxysporum & Watermelon & 2014 & NP \\
\hline Al7 & Abyek & Oxysporum & Watermelon & 2015 & NP \\
\hline A20 & Abyek & Proliferatum & Melon & 2015 & NP \\
\hline Q5 & Qazvin & Oxysporum & Tomato & 2013 & $\mathrm{P}$ \\
\hline Q6 & Qazvin & Oxysporum & Tomato & 2013 & $P$ \\
\hline Q8 & Qazvin & Oxysporum & Tomato & 2013 & NP \\
\hline Q10 & Qazvin & Oxysporum & Tomato & 2013 & $P$ \\
\hline Q13 & Qazvin & Oxysporum & Melon & 2014 & $\mathrm{P}$ \\
\hline Q19 & Qazvin & Solani & Melon & 2014 & NP \\
\hline Q20 & Qazvin & Proliferatum & Melon & 2014 & NP \\
\hline Q22 & Qazvin & Graminearum & Melon & 2015 & NP \\
\hline Q24 & Qazvin & Graminearum & Melon & 2015 & NP \\
\hline AL3 & Alvand & Oxysporum & Melon & 2013 & $\mathrm{P}$ \\
\hline AL4 & Alvand & Oxysporum & Melon & 2013 & $P$ \\
\hline ALI0 & Alvand & Oxysporum & Melon & 2014 & LP \\
\hline AL12 & Alvand & Oxysporum & Melon & 2014 & $\mathrm{P}$ \\
\hline AL18 & Alvand & Culmorum & Melon & 2015 & NP \\
\hline AL20 & Alvand & Proliferatum & Melon & 2015 & NP \\
\hline AVI & Avaj & Oxysporum & Melon & 2013 & $P$ \\
\hline AV2 & Avaj & Oxysporum & Melon & 2013 & P \\
\hline
\end{tabular}

Table 1: Contd....

\begin{tabular}{llllll}
\hline Isolatea Location & $\begin{array}{l}\text { Morphological } \\
\text { identification }\end{array}$ & $\begin{array}{c}\text { Year of } \\
\text { isolation }\end{array}$ \\
\hline AV5 & Avaj & Oxysporum & Melon & 2014 & $\mathrm{P}$ \\
AV6 & Avaj & Oxysporum & Melon & 2014 & $\mathrm{P}$ \\
AV7 & Avaj & Oxysporum & Watermelon & 2014 & $\mathrm{NP}$ \\
AV9 & Avaj & Oxysporum & Watermelon & 2015 & $\mathrm{NP}$ \\
\hline
\end{tabular}

Those isolates that were significantly different from the water control at $P=0.05$ (Dunnett's one-tailed $t$-test) in all experiments are considered $\mathrm{P}$; isolates not significantly different from controls are NP; and isolates were considered to have LP if it was significantly different than the control in some experiments but not others. P: Pathogenic, LP: Low pathogenicity, NP: Nonpathogenic

Fusarium graminearum, Fusarium proliferatum, and Fusarium solani. Identification based on morphological characteristics of our isolates was corroborated by comparison with sequences of their isolates in the GenBank.

\section{Pathogenicity Testing and Determination}

In the field and greenhouse, symptoms were include combinations of wilting, yellowing and chlorosis. Severely infected plants were wilted and died, but plants affected to a lesser degree became unproductive. In some cases, signs of decay on roots were observed. The most prominent internal symptom was vascular browning in plants. Symptoms of Fusarium wilt occurred after 37 days from the start. Reisolation was done from all the plants. Any signs of disease observed in the control plants.

All isolates were considered nonpathogenic (NP) in the experiment, when the $F$-test indicated no significant differences among our isolates and also control (Table 1). When our isolates indicated significant differences, $t$-test was used to compare all isolates with the control treatment, with $P=0.05$. Those isolates (at least two times of experiments) which were significantly higher DI than the control treatment were calculated pathogenic $(\mathrm{P})$. Those isolates which had a significant differences from the control treatment in few experiments but not all of the experiments, were calculated, low pathogenic (LP). 26 were identified as $F$. oxysporum and surveyed for pathogenicity on melon seedlings among of 50 Fusarium species that were gathered from melon and tomato in 2013. All of these were pathogenic in pathogenicity test on melon seedlings. In 2014, 40 Fusarium cultures were isolated from melon that 12 were identified as F.oxysporum and surveyed for pathogenicity. Six isolates of these isolates were pathogenic on melon plants. Furthermore, one isolate of F. solani and F. proliferatum identified that were NP. In 2015, 30 Fusarium cultures were isolated from melon that 9 isolates of $F$. oxysporum were collected from melon and watermelon were tested, and of these isolates, only three were pathogenic on melon plants. Seven F. oxysporum 
cultures isolated from watermelon in 2014 and 2015 (S4, S6, S9, A16, A17, AV7, AV9) were distinguished as non-pathogenic on melon seedlings. Moreover, three F. acuminatum were identified that all of them were LP. We had three F. graminearum cultures isolates from melon that all of them were non-pathogenic on melon seedlings (Table 1).

\section{Phylogenetic Analysis}

Phylogenetic tree from neighbor-joining showed progressively increasing resolution. Datasets were including five small groups of isolates (III-VIII) and two large groups (I, II) (Figure 1). Group (I) is resolved into smaller groups and groups (V-VIII) were found at the base of the tree (Figure 1). In clade (I), F. oxysporum from melon and watermelon grouped with NP (F14, F16, F22, F23, S4, S6, S9, AV7, AV9) from melon.

Moreover, 12 pathogenic F. oxysporum isolates from melon dissolved in clade (I) with a support value of $94 \%$; and one of LP F.oxysporum isolate (R27) situated with the pathogenic F. oxysporum isolates with $95 \%$ support. Clade (II) contained pathogenic F.oxysporum from Choobindar (K6, K7, K8, and K11), Danesfahan (D14, D15, D29, and D33) and Alvand (AL3, AL4, and AL12); LP F. oxysporum from Choobindar (K12), Danesfahan (D19) and Alvand (AL10). Group (III) (Qazvin F. oxysporum isolates Q5, Q6, Q10, and Q13) situated outside of two large groups (I and II). They were

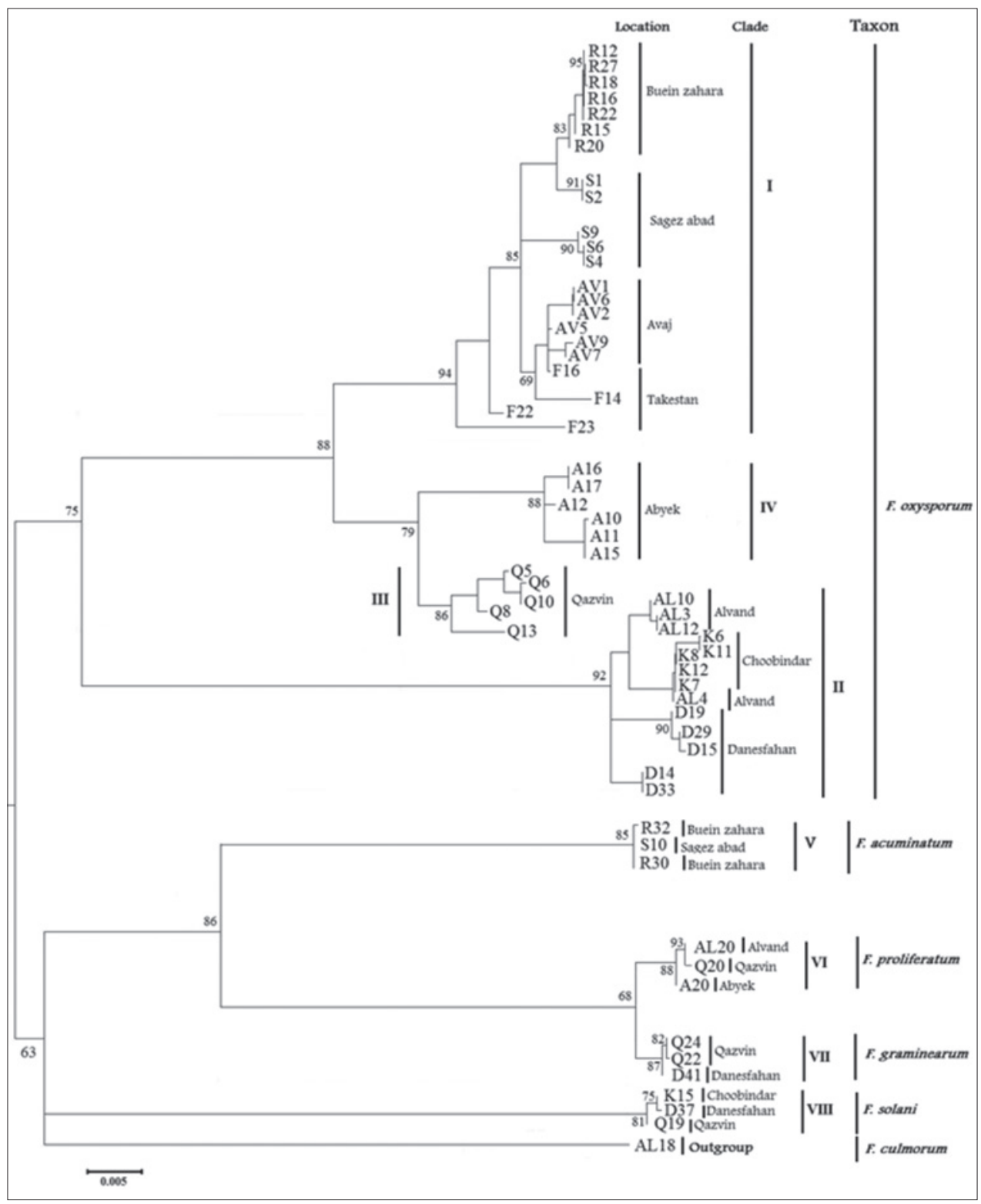

Figure 1: Neighbor-joining tree of 60 isolates of Fusarium oxysporum inferred from translation elongation factor $1 \alpha$ using Jukes-Cantor method. An isolate of $F$. culmorum was used as the out group 
all pathogenic on melon seedlings except Q8 that was NP. Clade (IV) contained pathogenic $F$. oxysporum from Abyek (A10, A11 and A12); LP F. oxysporum from Abyek (A15), and NP F.oxysporum from Abyek (A16 and A17 from watermelon plants).

Groups (V) (Buein zahra and Sagez abad F. acuminatum isolates R30, R32, and S10), (VI) (F. proliferatum isolates A20, Q20, and AL20), (VII) (Qazvin and Danesfahan F. graminearum isolates D41, Q22, and Q24) and (VIII) (F. solani isolates K15, D37, and Q19) also situated outside of two large groups (I and II) and in analyses were separated phylogenetically from isolates of $F$. oxysporum.

\section{DISCUSSION}

F. oxysporum is due to vascular wilt diseases in a large variety of important crops (Beckman, 1987). Fusarium can attack to vascular system of the plants and secrete its toxins, which cause the vascular wilting and then death within a few days or weeks (Champaco et al., 1993). Phylogenetic relationships in F. oxysporum have been studied by many researchers to determine evolutionary orientation among Fusarium species and to distinguish the dynamism in the different formae speciales (Gordon and Martyn, 1997). Using conserved gene regions in some of studies have showed that formae speciales of $F$. oxysporum are monophyletic, but some of other studies have distinguished polyphyletic groups (Koenig et al., 1997; Jimenez Gasco et al., 2002).

In this study, the phylogeny and the pathogenicity of F. oxysporum isolates were analyzed. The results indicated the genetic variability in Fusarium spp. from different locations in Qazvin Province. They indicated the genetic similarities and differences among the tested Fusarium spp. In this study, F.oxysporum strains from melon being placed in clades next and close to F. oxysporum strains from watermelon and tomato that show probably F. oxysporum from melon plants is polyphyletic. Clade (I) contained of pathogenic and NP isolates from Takestan, Buein zahra, Sagez abad, and Avaj. Phylogenetic analyses of $E F 1 \alpha$ sequences showed isolates in four major groups and four minor groups that all four major groups were $F$. oxysporum sequences from melon, tomato, and watermelon.

Neighbor-joining analysis of EF1 $\alpha$ with the highest support values demonstrate in the most clades (more than 70). The pathogenic and LP isolates are in clade (II) including isolates from Alvand, Choobindar, and Danesfahan. The pathogenic isolates are in clade (III) including isolates from Qazvin. The pathogenic, LP and NP isolates are in clade (IV) consisted isolates from Abyek. The isolates of F. solani, F. proliferatum, $F$ acuminatum, and $F$. graminearum evaluated form four clades separated each other which situate out of the four major clades. The identification isolates of $F$. oxysporum, F. solani, F. proliferatum, F. acuminatum, and F. graminearum by morphological methods supported with the results of the sequence of BLAST analysis.

The phylogenetic analyses indicated $F$. oxysporum from watermelon and tomato clustered with $F$. oxysporum from melon in most of clades. Furthermore, proofs for cross pathogenicity were recorded in pathogenicity tests where seven isolates of $F$. oxysporum from tomato used in this research were pathogenic on melon seedlings variety Minoo 095P. Many investigators have been reported pathogenic populations in the fungi (Koenig et al., 1997). However, genetic resistance may enable management in some locations, but some of the farmers have announced that managing of Fusarium wilt is decreased in different conditions. Although, they are ubiquitous but, the variability and pathogenicity of these isolates are rarely reported (Gordon and Okamoto, 1992).

A lot of studies of genetic diversity have been presented the possible correlation between genotypes and pathotypes (Nelson et al., 1997; Vakalounaki and Fragkiadakis, 1999). Variability in resistance in different locations shows races within $F$. oxysporum f. sp. melonis (Fom) (Jimenez-Gasco et al., 2002). It can propose races in Fom has been firmly-fixed on cross-pathogenicity among of isolates of melon, tomato and watermelon. However, in this study genetic variability and geographical locations had no obvious correlation for the F. oxysporum isolates. This is similar to a lack of correlation between the UP-PCR (by EF1 $\alpha$ ) and geographic location that is reported for F. oxysporum (Zhao et al., 2014). This is the opposite of a correlation observed between the RAPD pattern and geographic locations in a previous research study (Assigbetse et al., 1994).

Both in the field and greenhouse, the environmental conditions may participate diversity in pathogenicity response. Many of researchers found that inoculation quantity, plant variety, inoculation method, plant stage, and temperature can effect on the pathogenicity of Fom isolates. The cross pathogenicity of $F$. oxysporum isolates and their polyphyletic nature can be get the resistance breeding attempts much tough than other fungi. These findings would be required to assess tactics of resistance breeding in field or greenhouse conditions. 
This research study indicates the first molecular phylogenetic studies of $F$. oxysporum from melon in Qazvin province, Iran as compared to Zhao et al. (2014), which compared some species using EF1 $\alpha$ in Liaoning Province, China. This work supports the importance of expanding genetic markers to achieve a better perception of the evolutionary structure of fungi especially Fom. Furthermore, This study was include more geographical locations and similar numbers of isolates from each geographical location, so showed a more precise picture of aggregations between location origin, pathogenicity and phylogeny than other research studies.

\section{ACKNOWLEDGMENTS}

The author would like to thank the Agricultural Organization of Qazvin Province. This work is done with Personal expenses and without any help from Bu-Ali Sina University.

\section{REFERENCES}

Armstrong GM, Armstrong JK. Formae speciales and races of Fusarium oxysporum causing wilt diseases. In: Nelson PE, ToussounTA, Cook R, editors. Fusarium: Diseases, Biology and Taxonomy. Pennsylvania: The Pennsylvania State University Press, University Park; 1981. p. 391-9.

Assigbetse KB, Fernandez D, Dubois MP, Geiger JP. Differentiation of Fusarium oxysporum $\mathrm{f}$. sp. vasinfectum races on cotton by random amplified polymorphic DNA (RAPD) analysis. Phytopathology 1994;84:622-6.

Beckman CH. The Nature of Wilt Diseases of Plants. St. Paul, Minnesota, USA: APS Press; 1987. p. 175.

Booth C. The Genus Fusarium. Kew, London: Common Wealth Mycological Institute; 1971.

Champaco ER, Martyn RD, Miller ME. Comparison of Fusarium solani and F. oxysporum as causal agents of fruit net and root rot of muskmelons. Hortic Sci 1993;28:1174-7.

Dunnett CW. A multiple comparisons procedure for comparing several treatments with a control. J Am Stat Assoc 1955;50:1096-121.

Gordon TR, Martyn RD. The evolutionary biology of Fusarium oxysporum. Annu Rev Phytopathol 1997;35:111-28.

Gordon TR, Okamoto D, Jacobsen DJ. Colonization of muskmelon and non-susceptible crops by Fusarium oxysporum f. sp. melonis and other species of Fusarium. Phytopathology 1989;79:1095-100.

Gurjar G, Barve M, Giri A, Gupta V. Identification of Indian pathogenic races of Fusarium oxysporum f. sp. ciceris with gene specific, ITS and random markers. Mycologia 2009; 101:484-95.
Hanson LE, Hill AL. Fusarium species causing Fusarium yellows of sugar beet. J Sugar Beet Res 2004;41:163-78.

Harrow SA, Farrokhi-Nejad R, Pitman AR, Scott IA, Bentley A, Hide C, et al. Characterisation of New Zealand Fusarium populations using a polyphasic approach differentiates the $F$. avenaceum/F. acuminatum/F. tricinctum species complex in cereal and grassland systems. Fungal Biol 2010;114:293-311.

Hill AL, Reeves PA, Larson RL, Fenwick AL, Hanson LE, Panella L. Genetic variability among isolates of Fusarium oxysporum from sugar beet. Plant Pathol 2011;60:496-505.

Hsuan HM, Salleh B, Zakaria L. Molecular identification of Fusarium species in Gibberella fujikuroi species complex from rice, sugarcane and maize from Peninsular Malaysia. Int J Mol Sci 2011;12:6722-32.

Jimenez-Gasco MM, Milgroom MG, Jimenez- Diaz RM. Gene genealogies support Fusarium oxysporum f. sp. ciceris as a monophyletic group. Plant Pathol 2002;51:72-7.

Joshi M, Srivastava R, Sharma AK, Prakash A. Isolation and characterization of Fusarium oxysporum, a wilt causing fungus, for its pathogenic and non-pathogenic nature in tomato (Solanum lycopersicum). J Appl Nat Sci 2013;5:108-17.

Koenig RL, Ploetz RC, Kistler HC. Fusarium oxysporum f. sp. cubense consists of a small number of divergent and globally distributed clonal lineages. Phytopathology 1997;87:915-23.

Leslie JF, Summerell BA, Bullock S. The Fusarium Laboratory Manual. Oxford, UK: Blackwell Publishing Ltd.; 2006.

MatsumotoY, Ogawara T, Miyagi M, Watanabe N, Kuboyama T. Response of wild Cucumis species to inoculation with Fusarium oxysporum f. sp. melonis race 1,2y. J Jpn Soc Hortic Sci 2011;80:414-9.

Nelson AJ, Elias KS, Arévalo G E, Darlington LC, Bailey BA. Genetic characterization by RAPD analysis of isolates of Fusarium oxysporum f. sp. erythroxyli associated with an emerging epidemic in Peru. Phytopathology 1997;87:1220-5.

Nelson PE, Toussoun, TA, Marasas WFO. Fusarium Species: An Illustrated Manual for Identification. Pennsylvania: The Pennsylvania State University Press. University Park; 1983. p. 193.

Nirenberg H. Under structure about the morphological and biological differnzierung in Fusarium- Sektion Liseola. Mitteilungen aus der Biologischen Bundesansatlt fur Landund Forstwirtschaft. Vol. 169. Berlin, Dahlem: P.Parey; 1976. p. 11-7.

O’Donnell K, Cigelnik E, Nirenberg HI. Molecular systematics and phylogeography of the Gibberella fujikuroi species complex. Mycologia 1998;90:465-93.

Thompson JD, Gibson TJ, Plewniak F, Jeanmougin F, Higgins DG. The CLUSTAL_X windows interface: Fexible 
strategies for multiple sequence alignment aided by quality analysis tools. Nucleic Acids Res 1997;25:4876-82.

Vakalounaki DJ, Fragkiadakis GA. Genetic diversity of Fusarium oxysporum isolates from cucumber: Differentiation by pathogenicity, vegetative compatibility, and RAPD fingerprinting. Phytopathology 1999;89:161-8.

Windels CE. Fusarium. In: Singleton LL, Mihail JD,
Rush CM, editors. Methods for Research on Soilborne Phytopathogenic Fungi. St. Paul, MN, USA: APS Press; 1992. p. 115-25.

Zhao B, Yan J, Zhang S, Liu X, Gao Z. Phylogeny and pathogenicity of Fusarium spp. isolated from greenhouse melon soil in Liaoning province. Saudi J Biol Sci 2014;21:374-9. 